\title{
Comparison of Disease-Modifying Therapies for the Management of Multiple Sclerosis: Analysis of Healthcare Resource Utilization and Relapse Rates from US Insurance Claims Data
}

\author{
Jacqueline Nicholas ${ }^{1} \cdot$ Aaron Boster ${ }^{1} \cdot \mathrm{Ning}_{\mathrm{Wu}^{2}} \cdot$ Wei-Shi $\mathrm{Yeh}^{2} \cdot$ Monica Fay $^{2} \cdot$ \\ Jon Kendter ${ }^{2} \cdot$ Ming-Yi Huang ${ }^{2} \cdot$ Andrew Lee ${ }^{2}$
}

Published online: 9 August 2017

(c) The Author(s) 2017. This article is an open access publication

\begin{abstract}
Background Data on comparative healthcare resource utilization and costs associated with the newer oral diseasemodifying therapies (DMTs) for managing relapsingremitting multiple sclerosis (MS) in routine clinical practice are limited. The purpose of this study was to estimate healthcare resource utilization, costs, and relapse rates in the year after initiating treatment with dimethyl fumarate (DMF), interferon (IFN)- $\beta$, glatiramer acetate (GA), teriflunomide, or fingolimod in routine clinical practice for patients with MS who did not receive a DMT in the previous year.

Methods Patients initiating DMF, IFN $\beta$, GA, teriflunomide, or fingolimod were identified based on claims data from 2012 to 2015 in the Truven MarketScan Commercial Claims Databases $(n=4194)$. Healthcare resource utilization assessment included the proportion of patients who were hospitalized, or had emergency room (ER) or urgent care (UC) visits. Healthcare costs were estimated for 1 year before and 1 year after DMT initiation. Relapse episodes were identified based on a published claims-based algorithm and clinical input from the research investigators.
\end{abstract}

Electronic supplementary material The online version of this article (doi:10.1007/s41669-017-0035-2) contains supplementary material, which is available to authorized users.

Andrew Lee

andrew.lee@biogen.com

1 OhioHealth Multiple Sclerosis Center, Riverside Methodist Hospital, Columbus, OH, USA

2 Biogen, 225 Binney Street, Cambridge, MA 02142, USA
Results After DMT initiation, significant reductions in the proportions of patients who were hospitalized or requiring ER/UC visits were observed in all patient cohorts $(p<0.001$ and $p<0.05$, respectively). Non-prescription medical costs decreased after DMT initiation, with the largest decrease observed in the DMF cohort (US\$5761 reduction, $p<0.0001)$. Reductions in non-prescription medical costs were associated with decreased use of outpatient services and inpatient hospital stays, and have the potential to partially offset DMT costs.

Conclusions DMT initiation is associated with reductions in healthcare resource utilization and non-prescription medical costs in routine clinical practice.

\section{Key Points for Decision Makers}

An analysis of patients receiving disease-modifying therapies (DMTs) in routine clinical practice from US claims data indicate that DMTs provide reductions in healthcare resource utilization and medical costs that have the potential to partially offset DMT costs.

Non-prescription medical costs decreased after DMT initiation, with the largest decrease observed in the dimethyl fumarate cohort (US\$5761 reduction, $p<0.0001)$. Non-prescription medical cost reductions were predominantly driven by decreased use of outpatient services and by a reduction in inpatient hospital stays.

Further research is required to determine the longterm impact of DMT initiation on resource utilization and costs in routine clinical practice. 


\section{Introduction}

Multiple sclerosis (MS) is an incurable, chronic autoimmune disease of the central nervous system, characterized by inflammation, demyelination, and axonal degeneration, and the resultant loss of neurological function [1-3]. MS is a leading cause of non-traumatic neurological disability in young adults, affecting approximately 2.3 million individuals worldwide and more than 400,000 individuals in the US $[4,5]$. Approximately $85 \%$ of people with MS have relapse-remitting MS (RRMS), characterized by neurological relapses interspersed with periods of clinical remission; however, silent damage continues to occur and is often detected on neuroimaging [6].

The socioeconomic burden of MS is significant, with recent records estimating MS to be the second most expensive chronic healthcare condition to manage after congestive heart failure [7]. In a review of mean annual costs associated with MS in the US, total indirect and direct healthcare costs ranged from US $\$ 8528$ to US $\$ 54,244$ per patient per year [7]. Indirect costs associated with MS are also significant; approximately $56-58 \%$ of individuals with MS are unemployed [8], and the average total annual indirect costs for employees with MS are US $\$ 4352$ higher than in the general working population [9].

Disease-modifying therapies (DMTs) are a key component of managing patients with MS, providing reductions in relapse rates and magnetic resonance imaging lesion accumulation, and having a demonstrated association with improvements in clinical and quality-of-life outcomes $[10,11]$. Recent availability of oral DMTs (dimethyl fumarate [DMF], teriflunomide, and fingolimod) has provided healthcare professionals and patients with alternative treatment options to the injectable DMTs (interferon [IFN]$\beta$ and glatiramer acetate [GA]) for the management of MS. Evidence derived from clinical trials, meta-analyses, and indirect comparisons suggests that the oral therapies provide at least similar efficacy to IFN $\beta$ and GA, and that DMF and fingolimod may provide superior efficacy compared with teriflunomide $[12,13]$.

However, therapeutic outcomes in routine clinical practice may potentially be influenced by factors that are controlled for during clinical trials, such as prior disease history, treatment history, disease duration, and disease severity $[14,15]$. The evaluation of medical reimbursement claims data is therefore critical for assessing therapeutic effectiveness, resource utilization, and associated healthcare costs for available DMTs in the real world. Studies of real-world effectiveness for available DMTs in routine clinical practice suggest that fingolimod and DMF have similar effectiveness in reducing relapse rates, and greater effectiveness than IFN $\beta$, GA, and teriflunomide $[16,17]$.
Moreover, increased healthcare costs associated with DMT initiation may be partially offset by a reduction in nonprescription medical costs and healthcare resource utilization in individuals with MS $[18,19]$. For example, a retrospective administration claims analysis demonstrated that natalizumab therapy in patients who were DMT-naive compared with those who had previously received DMT treatment led to a significant reduction in the number of inpatient stays ( 7.6 vs. $2.4 \%$, respectively, $p<0.001$ ) and associated costs (median US\$12,078 vs. US\$9784, $p<0.001$ ) [18]. Similarly, a budget impact analysis for DMF from a Canadian health system perspective demonstrated a partial offsetting of increased total healthcare costs on DMT initiation due to a reduction in costs associated with disease relapse [19]. However, data comparing healthcare resource utilization, costs, and patient outcomes for the newer oral DMTs in routine clinical practice are currently limited.

The objectives of this study were to estimate healthcare resource utilization and costs, and assess annualized relapse rates (ARRs), for patients with MS who did not receive a DMT in the previous year and who initiated DMF, IFN $\beta$, $\mathrm{GA}$, teriflunomide, or fingolimod in routine clinical practice.

\section{Methods}

\subsection{Data Source}

This retrospective claims analysis was based on data from the Truven MarketScan Commercial Claims Databases from January 2012 to June 2015, inclusive. These databases contain the de-identified administrative claims and eligibility records of over 120 million commercially insured individuals from all US regions, and include three main components: (1) claims for medical services provided in both inpatient and outpatient settings with the associated diagnosis, service dates, place of service, procedures performed, and patient- and plan-paid amounts; (2) pharmacy claims with associated dispensed date, National Drug Code, dose, days of supply, and patient- and plan-paid amounts; and (3) patient demographic information, including year of birth, sex, health plan type, and region of residence. Data on MS diagnosis criteria, disease severity and duration, disease progression, laboratory or imaging test results, or measurements of neurologic or MS-related disability, such as the Expanded Disability Status Scale (EDSS), are not routinely captured in claims data and were therefore not available for inclusion in the present analysis.

All patient information was anonymized, and patient confidentiality was maintained through compliance with Health Insurance Portability and Accountability Act 
regulations. The analysis presented is based on previously collected data and does not involve any new studies of human or animal subjects performed by any of the authors.

\subsection{Patient Identification}

Adult patients with MS were identified if they had at least one hospitalization with an associated diagnosis suggesting MS (International Classification of Diseases, Ninth Revision, Clinical Modification [ICD-9-CM] 340) or two outpatient visits with ICD-9-CM 340 and dated at least 30 days apart. Included patients may have had any MS subtype and did not necessarily have relapsing-remitting disease. Included patients had initiated treatment with DMF, IFN $\beta$, GA, teriflunomide, or fingolimod during the period January 2013 to June 2014, inclusive. Initiation was defined as having no claims for the same DMT during the previous year. Patients with claims for any DMT during the year prior to initiation were not included in the analysis; data were not available to confirm whether patients had received a DMT prior to the 1-year pre-index period, or whether treatment was given as first, second, or subsequent line of therapy. DMTs were initiated according to the treating physician's guidance and commercial insurance plan restrictions, as per routine clinical practice.

Eligible DMT initiators were assigned to corresponding DMT cohorts according to the DMT initiated, and the date of DMT initiation was set as the index date. Patients aged between 18 and 64 years at the index date were included in the analysis. To ensure complete data for the assessment of baseline characteristics and study outcomes, included patients were required to have at least 1 year of continuous enrollment before and 1 year after the index date in a participating commercial insurance plan (Fig. 1).

\subsection{Study Measures}

Patient demographics included age at index date, sex, type of health plan enrolled, and region of residence. Baseline clinical characteristics were assessed based on medical and pharmacy claims dated within 1 year prior to the index date. Chronic comorbidities were measured using the Charlson Comorbidity Index (CCI) [20], a composite score calculated based on the presence of 22 chronic conditions (e.g. diabetes, peptic ulcer, liver disease, cancer) and widely used to assess chronic disease burden; higher scores are indicative of an increased burden of chronic disease. The proportion of patients experiencing MS-related symptoms in the 1 year prior to the index date was estimated. The selection of MS-related symptoms was based on a published study [21]; selected symptoms were critically reviewed and ratified by the research investigators.

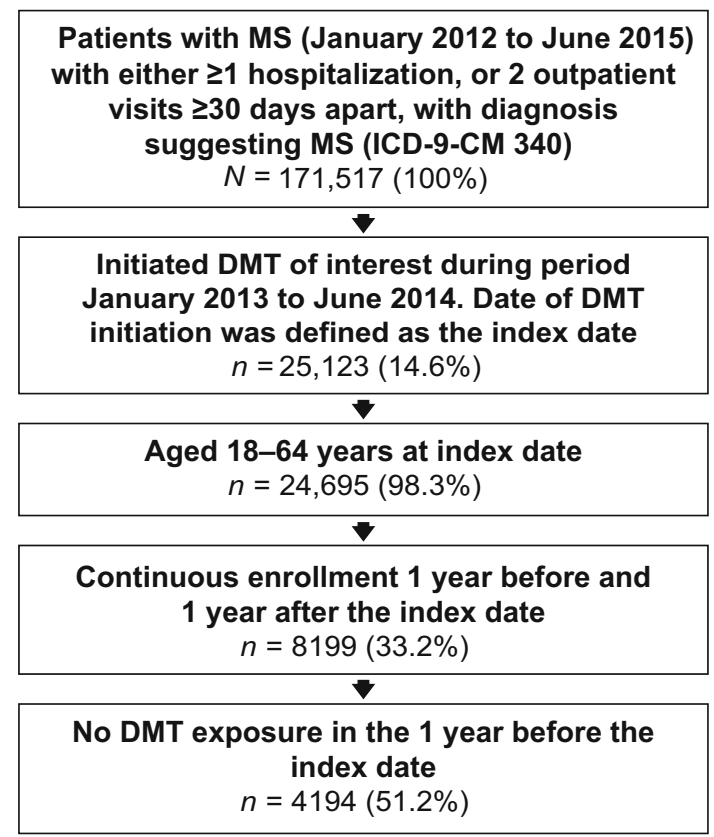

Fig. 1 Patient selection process. DMT disease-modifying therapy, ICD-9-CM International Classification of Diseases, Ninth Revision, Clinical Modification, $M S$ multiple sclerosis

Healthcare resource utilization was defined as the proportion of patients with MS who were hospitalized or had emergency room (ER) or urgent care (UC) visits during the 1 year prior to and 1 year following the index date. Total healthcare costs and costs by settings (i.e. inpatient, outpatient [including occupational therapy, physical therapy, and durable medical equipment], ER/UC, and pharmacy costs) were calculated. Medical costs excluded the cost of prescriptions. All costs were adjusted to 2014 US dollar (US\$) values based on the Consumer Price Index Medical Component [22]. DMT costs were also assessed and comprised plan and patient payments for filled prescriptions. Included patients had only pharmacy claims for an eligible DMT; patients with both medical and pharmacy claims for an eligible DMT were excluded from the analysis to reduce the risk of double-counting DMT costs and because medical DMT claims do not include information on days of supply, compliance, or duration of DMT use.

ARR was defined as the number of relapse episodes that a patient had during either the year before or the year after the index date. A published claims-based algorithm was used to identify relapse episodes [23, 24]. A moderate-tosevere relapse was defined as hospitalization with a primary diagnosis suggesting MS, or an outpatient visit with at least one associated diagnosis suggesting MS together with evidence of receiving high-dose oral corticosteroid ( $\geq 500 \mathrm{mg} /$ day prednisolone or equivalent), intravenous corticosteroid, corticotropin (i.e. adrenocorticotropic hormone), or plasma exchange within 30 days of the 
outpatient visit. All qualifying hospitalizations or outpatient visits that occurred during a single 30 -day period were considered as one relapse episode to account for the potential for multiple clinical encounters within a single relapse episode. For multiple qualifying hospitalizations and outpatient visits, provided that each successive visit occurred within 30 days of the previous visit, events were amalgamated into one relapse episode. For example, three hospitalizations or outpatient visits, each 25 days apart, would be considered as a single relapse episode.

\subsection{Statistical Analysis}

Analysis of variance (continuous variables and counts) and Chi square tests (categorical variables) were used to detect significant differences across cohorts. Paired $t$ tests (costs) and the McNemar test (any hospitalization or UC visit) were used to detect significant changes over time in ARR, healthcare resource utilization, and costs for each cohort during the 1 year prior to and 1 year following the index date.

Poisson regression was used to compare the ARRs during the year after DMT initiation between DMT cohorts. Confounders included in the models comprised age (18-30, 31-40, 41-50, and 51-64 years), sex, region of residence, number of relapses during the year before the index date $(0,1,2$, and $\geq 3)$, CCI score, and the presence of individual MS-related symptoms (Table 1). As the largest cohort included in the study, the DMF cohort was assigned as the reference group. The adjusted incidence rate ratio (IRR) for relapse relative to the DMF cohort is presented with the associated 95\% confidence interval (CI). For example, an IRR of 1.10 indicates that the ARR in the year after DMT initiation is $10 \%$ higher in the DMT cohort assessed relative to the DMF cohort.

A generalized linear model (GLM) with a log link and a gamma distribution was used to compare cost data; ARR data were compared using a GLM with a log link and a Poisson distribution. Difference-in-difference (DiD) regression analysis was used to compare changes in ARRs and total non-prescription medical costs during the 1 year prior to and 1 year following the index date between cohorts, and was adjusted for age, sex, region of residence, CCI (categorized as $0,1,2$, and $\geq 3$ ), and MS-related symptoms (DiD model parameters included in the Online Resources). DiD regression is a statistical technique developed to control for unobserved or undefined confounders in observational data [25]. It provides advantages over alternative methods for controlling for baseline data values because it can control for unobserved heterogeneity in the dataset over the study period [25]. In our regression analysis, we cannot adjust for confounders that cannot be captured, or are inadequately captured, in claims data (e.g. duration of MS, severity of MS). Using DiD analysis therefore enables us to control for unmeasured baseline differences between DMT cohorts. DiD was used for the primary analysis of the ARRs and total non-prescription medical cost data. Details of the regression models are included in the accompanying Online Resources.

\section{Results}

Data from 4194 patients were included in the analysis. Demographic and clinical baseline characteristics for all included patients are shown in Table 1 . Of the included patients, $34.5 \%$ had received DMF, $23.1 \%$ had received IFN $\beta, 29.9 \%$ had received GA, 5.4\% had received teriflunomide, and $7.1 \%$ had received fingolimod. Mean (standard deviation) age ranged from 43.5 (10.5) years for GA to 48.7 (9.6) years for teriflunomide (Table 1), and differed significantly across the cohorts $(p<0.05)$. The proportion of female patients ranged from $76.5 \%$ (DMF) to $82.2 \%$ (teriflunomide); the proportion of female patients in the DMF cohort did not differ significantly from the other DMT cohorts. Patient geographical distribution was generally consistent across DMT cohorts. The presence of MSrelated symptoms differed across cohorts, with significant differences observed between the DMF cohort and the other DMT cohorts (Table 1). Compared with the DMF cohort, mean CCI score was significantly lower in the fingolimod cohort $(p<0.05)$ and significantly greater in the GA and teriflunomide cohorts $(p<0.05)$.

\subsection{Healthcare Resource Utilization}

The proportion of patients who were hospitalized was significantly lower in the year after DMT initiation than in the year before initiation for all DMT cohorts (Table 2; $p<0.001$ for all comparisons). Reductions in the proportion of patients who were hospitalized ranged from $3.1 \%$ for teriflunomide to $10.1 \%$ for IFN $\beta$. The proportion of patients requiring an ER/UC visit was significantly lower in the postindex period for all cohorts (Table 2; $p<0.05$ for fingolimod, and $p<0.0001$ for all other DMT comparisons). Reductions in the proportion of patients with an ER/UC visit ranged from $0.7 \%$ for fingolimod to $12.6 \%$ for GA.

\subsection{Costs}

Table 3 provides cost data for all patient cohorts during the 1 year prior to and 1 year following the index date. Overall healthcare costs increased for all cohorts in the 1-year postindex period. Increases were lowest for the DMF cohort (US\$40,920) and highest for the fingolimod cohort (US\$53,089). DMT costs were the largest driver of postindex period costs, ranging from US $\$ 42,652$ for teriflunomide to US\$57,581 for fingolimod. 
Table 1 Baseline demographic and clinical characteristics

\begin{tabular}{|c|c|c|c|c|c|}
\hline & \multicolumn{5}{|l|}{ DMT } \\
\hline & $\begin{array}{l}\text { Dimethyl fumarate } \\
{[n=1447]}\end{array}$ & $\begin{array}{l}\text { Interferon- } \beta \\
{[n=969]}\end{array}$ & $\begin{array}{l}\text { Glatiramer acetate } \\
{[n=1254]}\end{array}$ & $\begin{array}{l}\text { Teriflunomide } \\
{[n=225]}\end{array}$ & $\begin{array}{l}\text { Fingolimod } \\
{[n=299]}\end{array}$ \\
\hline Age, years [mean (SD)] & $45.4(9.9)$ & $43.9(10.8)^{\mathrm{a}}$ & $43.5(10.5)^{\mathrm{a}}$ & $48.7(9.6)^{\mathrm{a}}$ & $43.9(9.9)^{\mathrm{b}}$ \\
\hline Female patients, $\%$ patients & 76.5 & 76.8 & 78.7 & 82.2 & 76.9 \\
\hline \multicolumn{6}{|l|}{ Region } \\
\hline Northeast & 25.2 & 20.7 & 25.3 & 19.6 & 21.4 \\
\hline Midwest & 24.3 & 27.3 & 20.7 & 28.9 & 28.8 \\
\hline South & 31.6 & 36.1 & 34.6 & 34.7 & 35.1 \\
\hline West & 17.8 & 15.0 & 18.3 & 15.1 & 13.7 \\
\hline Unknown & 1.1 & 0.8 & 1.0 & 1.8 & 1.0 \\
\hline \multicolumn{6}{|l|}{ Health plan type, $\%$ patients } \\
\hline HMO & 11.1 & 13.1 & 10.2 & 10.2 & 10.0 \\
\hline POS & 7.1 & 6.7 & 6.0 & 4.4 & 6.4 \\
\hline PPO & 58.8 & 57.9 & 62.6 & 64.9 & 57.9 \\
\hline CHDP & 12.2 & 11.2 & 10.4 & 11.6 & 14.4 \\
\hline Other & 10.9 & 11.0 & 10.8 & 8.9 & 11.4 \\
\hline \multicolumn{6}{|l|}{ CCI score } \\
\hline Mean (SD) & $0.63(1.19)$ & $0.69(1.26)$ & $0.78(1.34)^{\mathrm{a}}$ & $0.85(1.45)^{\mathrm{b}}$ & $0.48(1.05)^{\mathrm{b}}$ \\
\hline $0, \%$ patients & 70.0 & $69.2^{\mathrm{a}}$ & $65.6^{\mathrm{b}}$ & $61.3^{\mathrm{b}}$ & 76.9 \\
\hline $1, \%$ patients & 10.7 & 7.4 & 10.6 & 15.6 & 9.7 \\
\hline $2, \%$ patients & 12.0 & 15.7 & 14.3 & 12.0 & 7.0 \\
\hline$\geq 3, \%$ patients & 7.3 & 7.6 & 9.6 & 11.1 & 6.4 \\
\hline Any relapse in 1 year prior to index date, $\%$ patients & 31.7 & 28.6 & 26.6 & 28.9 & 32.1 \\
\hline \multicolumn{6}{|l|}{ MS-related symptoms, $\%$ patients } \\
\hline Other causes of myelitis & 2.0 & 3.1 & $4.1^{\mathrm{a}}$ & 0.4 & 1.3 \\
\hline Unspecified CNS demyelinating disease & 12.6 & $19.8^{\mathrm{a}}$ & $24.8^{\mathrm{a}}$ & 13.3 & 9.0 \\
\hline Disorders of optic nerves and visual pathways & 11.3 & 12.9 & 13.6 & 9.3 & 10.4 \\
\hline Neurogenic bladder NOS & 5.9 & $2.7^{\mathrm{a}}$ & $2.6^{\mathrm{a}}$ & 7.1 & 3.7 \\
\hline Other soft tissue disorders & 2.5 & 3.4 & 3.3 & 2.2 & 1.3 \\
\hline Dizziness or giddiness & 10.4 & $14.7^{\mathrm{a}}$ & $16.2^{\mathrm{a}}$ & 8.9 & $6.7^{\mathrm{b}}$ \\
\hline Fatigue or malaise & 27.9 & 24.9 & 28.1 & 29.8 & 25.8 \\
\hline
\end{tabular}

$C C I$ Charlson Comorbidity Index, CHDP Child Health and Disability Prevention, CNS central nervous system, $D M T$ disease-modifying therapy, $H M O$ Health Maintenance Organization, $M S$ multiple sclerosis, $N O S$ not otherwise specified, $P O S$ point-of-service, $P P O$ preferred provider organization, $S D$ standard deviation

${ }^{\text {a }}$ Pairwise comparison versus dimethyl fumarate significantly different at $p<0.01$

b Pairwise comparison versus dimethyl fumarate significantly different at $p<0.05$

Unadjusted non-prescription medical costs were significantly lower in the post-index period than the pre-index period for the DMF cohort $(p<0.0001)$, the IFN $\beta$ cohort $(p<0.01)$, and the GA and fingolimod cohorts (both comparisons $p<0.05$ ) (Fig. 2; Table 3); the US $\$ 1437$ cost reduction for teriflunomide in the post-index period was not statistically significant $(p=0.385)$. The largest decrease in non-prescription medical costs was observed in the DMF cohort (-US\$5761, $p<0.0001$ ).

DiD analysis on unadjusted non-prescription medical cost reductions demonstrated that DMF provided a significantly greater cost reduction than $\operatorname{IFN} \beta(p<0.01)$, GA $(p<0.0001)$, and teriflunomide $(p<0.01)$. After adjusting for confounders, DiD analysis indicated that DMF provided a significantly greater reduction in medical costs than IFN $\beta$ and teriflunomide (both comparisons $p<0.01)$ and GA $(p<0.001)$. No significant difference in the reduction of total non-prescription medical costs was observed between the DMF and fingolimod cohorts $(p=0.7553)$.

Medical cost reductions (excluding prescription medicine costs) in the post-index period were driven largely by cost reductions for outpatient services and hospitalizations (Table 3). Mean cost reductions for outpatient services and hospitalizations were greatest for the DMF cohort (-US\$4395 and -US\$1082, respectively). 
Table 2 Percentage of patients with hospitalizations or emergency room/urgent care visits 1 year before and 1 year after the index date for patients with MS and no DMT exposure during the year before the index date

\begin{tabular}{|c|c|c|c|c|c|}
\hline & \multicolumn{5}{|l|}{ DMT } \\
\hline & $\begin{array}{l}\text { Dimethyl fumarate } \\
{[n=1447]}\end{array}$ & $\begin{array}{l}\text { Interferon- } \beta \\
{[n=969]}\end{array}$ & $\begin{array}{l}\text { Glatiramer acetate } \\
{[n=1254]}\end{array}$ & $\begin{array}{l}\text { Teriflunomide } \\
{[n=225]}\end{array}$ & $\begin{array}{l}\text { Fingolimod } \\
{[n=299]}\end{array}$ \\
\hline \multicolumn{6}{|l|}{ Hospitalization, $\%$ patients } \\
\hline 1 year before index date & 14.1 & 17.1 & 17.3 & 13.8 & 13.4 \\
\hline 1 year after index date & 7.0 & 7.0 & 7.6 & 10.7 & 5.7 \\
\hline$p$ value (pre-post comparison) & $<0.0001$ & $<0.0001$ & $<0.0001$ & $<0.0001$ & 0.0005 \\
\hline \multicolumn{6}{|c|}{ Emergency room/urgent care visits, $\%$ patients } \\
\hline 1 year before index date & 30.7 & 30.7 & 35.2 & 30.2 & 25.1 \\
\hline 1 year after index date & 25.1 & 21.8 & 22.6 & 23.6 & 24.4 \\
\hline$p$ value (pre-post comparison) & $<0.0001$ & $<0.0001$ & $<0.0001$ & $<0.0001$ & 0.0169 \\
\hline
\end{tabular}

$D M T$ disease-modifying therapy, $M S$ multiple sclerosis

Table 3 Mean costs in US\$ for total non-prescription medical, drug, and total healthcare 1 year prior to and 1 year following the index date for patients with MS and no DMT exposure in the year prior to the index date

\begin{tabular}{|c|c|c|c|c|c|c|c|c|c|c|}
\hline & \multicolumn{10}{|l|}{ DMT } \\
\hline & \multicolumn{2}{|c|}{$\begin{array}{l}\text { Dimethyl fumarate } \\
{[n=1447]}\end{array}$} & \multicolumn{2}{|c|}{$\begin{array}{l}\text { Interferon- } \beta \\
{[n=969]}\end{array}$} & \multicolumn{2}{|c|}{$\begin{array}{l}\text { Glatiramer acetate } \\
{[n=1254]}\end{array}$} & \multicolumn{2}{|c|}{$\begin{array}{l}\text { Teriflunomide } \\
{[n=225]}\end{array}$} & \multicolumn{2}{|c|}{$\begin{array}{l}\text { Fingolimod } \\
{[n=299]}\end{array}$} \\
\hline & $\begin{array}{l}\text { Pre- } \\
\text { index }\end{array}$ & $\begin{array}{l}\text { Post- } \\
\text { index }\end{array}$ & $\begin{array}{l}\text { Pre- } \\
\text { index }\end{array}$ & $\begin{array}{l}\text { Post- } \\
\text { index }\end{array}$ & $\begin{array}{l}\text { Pre- } \\
\text { index }\end{array}$ & $\begin{array}{l}\text { Post- } \\
\text { index }\end{array}$ & $\begin{array}{l}\text { Pre- } \\
\text { index }\end{array}$ & $\begin{array}{l}\text { Post- } \\
\text { index }\end{array}$ & $\begin{array}{l}\text { Pre- } \\
\text { index }\end{array}$ & $\begin{array}{l}\text { Post- } \\
\text { index }\end{array}$ \\
\hline \multirow{2}{*}{$\begin{array}{l}\text { Total non-prescription medical } \\
\text { costs }\end{array}$} & 16,853 & 11,093 & 13,669 & 11,087 & 14,623 & 12,405 & 14,992 & 13,555 & 17,508 & 12,593 \\
\hline & \multicolumn{2}{|c|}{$\Delta-5761^{\mathrm{a}}$} & \multicolumn{2}{|c|}{$\Delta-2582^{\mathrm{a}}$} & \multicolumn{2}{|c|}{$\Delta-2219^{\mathrm{a}}$} & \multicolumn{2}{|c|}{$\Delta-1437$} & \multicolumn{2}{|c|}{$\Delta-4915^{\mathrm{a}}$} \\
\hline \multirow[t]{2}{*}{ Inpatient stays } & 3217 & 2135 & 3217 & 2378 & 3493 & 3380 & 3667 & 3351 & 4526 & 3816 \\
\hline & \multicolumn{2}{|c|}{$\Delta-1082$} & \multicolumn{2}{|c|}{$\Delta-839$} & \multicolumn{2}{|c|}{$\Delta-113$} & \multicolumn{2}{|c|}{$\Delta-316$} & \multicolumn{2}{|c|}{$\Delta-710$} \\
\hline \multirow[t]{2}{*}{ ER visits } & 1010 & 726 & 1089 & 590 & 1054 & 576 & 807 & 631 & 626 & 678 \\
\hline & \multicolumn{2}{|l|}{$\Delta-284$} & \multicolumn{2}{|c|}{$\Delta-499$} & \multicolumn{2}{|c|}{$\Delta-478$} & \multicolumn{2}{|c|}{$\Delta-176$} & \multicolumn{2}{|l|}{$\Delta+51$} \\
\hline \multirow[t]{2}{*}{ Outpatient services } & 12,626 & 8232 & 9364 & 8119 & 10,076 & 8448 & 10,518 & 9573 & 12,355 & 8099 \\
\hline & \multicolumn{2}{|c|}{$\Delta-4395$} & \multicolumn{2}{|c|}{$\Delta-1244$} & \multicolumn{2}{|c|}{$\Delta-1628$} & \multicolumn{2}{|c|}{$\Delta-945$} & \multicolumn{2}{|c|}{$\Delta-4256$} \\
\hline \multicolumn{11}{|l|}{ Drug costs } \\
\hline \multirow[t]{2}{*}{ DMT } & 0 & 46,252 & 0 & 47,889 & 0 & 47,633 & 0 & 42,652 & 0 & 57,581 \\
\hline & \multicolumn{2}{|c|}{$\Delta+46,252$} & \multicolumn{2}{|c|}{$\Delta+47,889$} & \multicolumn{2}{|c|}{$\Delta+47,633$} & $\Delta+42$ & & $\Delta+57$ & \\
\hline Non-DMT & 3959 & 4386 & 2738 & 3740 & 2698 & 3593 & 5803 & 6763 & 4249 & 4672 \\
\hline & $\Delta+428$ & & $\Delta+10$ & & $\Delta+89$ & & $\Delta+96$ & & $\Delta+42$ & \\
\hline Total healthcare costs & 20,812 & 61,731 & 16,407 & 62,716 & 17,321 & 63,630 & 20,795 & 62,970 & 21,756 & 74,846 \\
\hline & $\Delta+40,9$ & & $\Delta+46$ & & $\Delta+46$ & & $\Delta+42$ & & $\Delta+53$ & \\
\hline
\end{tabular}

$D M T$ disease-modifying therapy, ER emergency room, $M S$ multiple sclerosis

a Significant difference in cost between pre-index period and post-index period $(p<0.05)$

\subsection{Annualized Relapse Rates}

Unadjusted ARRs for the year prior to the index date ranged from 0.31 for GA to 0.43 for DMF (Fig. 3). The ARR in the year before initiation was significantly greater in the DMF cohort than in the GA and IFN $\beta$ cohorts ( $p<0.001$ and $p<0.05$, respectively). Significant reductions in unadjusted ARR were observed in the DMF (0.14 reduction, $p<0.0001)$ and fingolimod (0.11 reduction, $p<0.05)$ cohorts in the year after initiating therapy. ARR reductions in the post-index period for patients in the IFN $\beta$, $\mathrm{GA}$, and teriflunomide cohorts were not statistically significant $(p=0.5940, \quad p=0.3748, \quad$ and $p=0.7822$, respectively).

DiD analysis on unadjusted ARRs demonstrated that change of ARR in the 1 year following initiation of 




Year prior to the index date Year after the index date

Fig. 2 Differences in non-prescription medical costs $^{1}$ for 1 year before and 1 year after the index date for patients with multiple sclerosis and no DMT exposure during the year before the index date. ${ }^{1}$ Difference-in-difference analysis adjusted for confounders indicated that reduction of total non-prescription medical costs in the 1 year following DMT initiation versus the prior year were statistically significantly greater in the dimethyl fumarate cohort versus the interferon- $\beta \quad(p=0.0012)$, glatiramer acetate $(p=0.0003)$, and teriflunomide $(p=0.005)$ cohorts. No significant differences in reduction in total non-prescription medical costs were observed between the dimethyl fumarate and fingolimod cohorts $(p=0.7553)$. $D M T$ disease-modifying therapy
Fig. 3 Annualized relapse rates for 1 year before and 1 year after the index date for patients with multiple sclerosis and no disease-modifying therapy exposure during the year before the index date

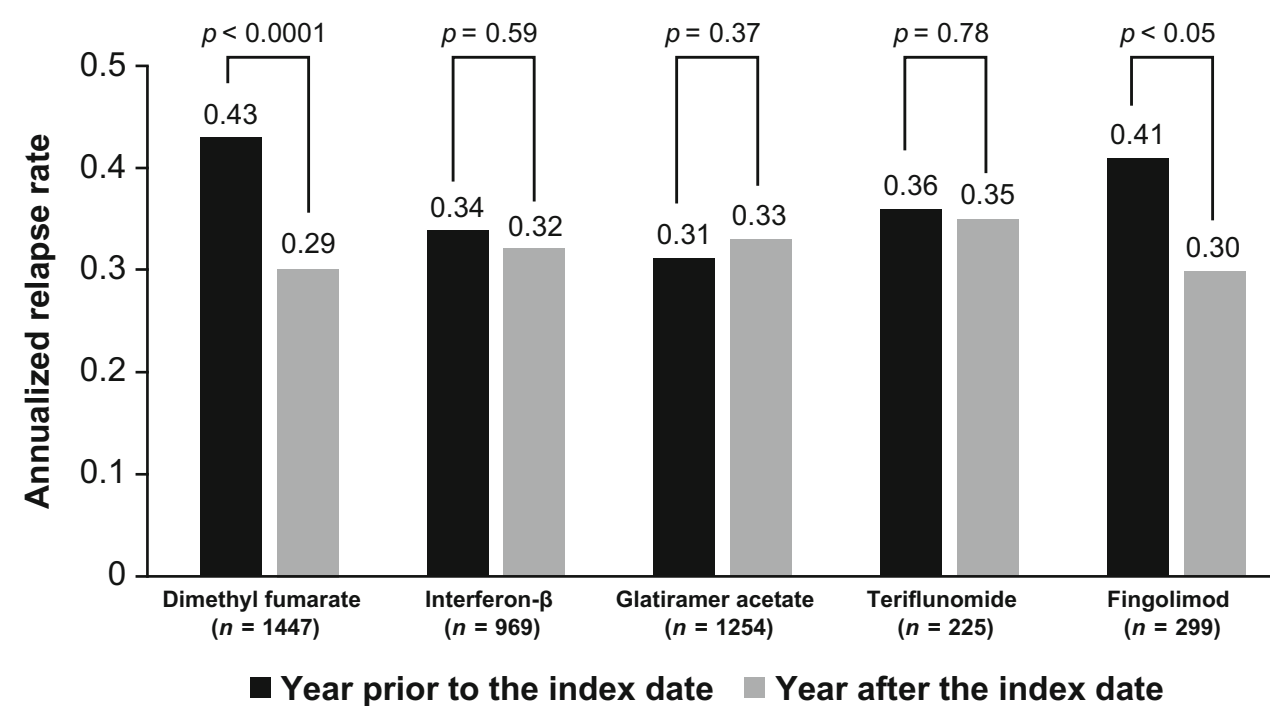

the DMF cohort (Online Resource Table S1). Adjusted IRRs compared with DMF were $1.23(1.06-1.43)$ for IFN $\beta, 1.27(1.10-1.46)$ for GA, $1.28(1.00-1.63)$ for teriflunomide, and $1.07(0.85-1.35)$ for fingolimod. Adjusted IRRs were significantly higher in the IFN $\beta$, GA, and teriflunomide cohorts than the DMF cohort $(p<0.05$ for all comparisons). Patient age $(p<0.05)$, number of relapses in the year before DMT initiation $(p<0.0001)$, and the presence of fatigue or malaise $(p<0.05)$ were significant predictors for ARR after DMT initiation. 


\section{Discussion}

In this retrospective study of real-world healthcare resource utilization and cost data for oral and injectable DMTs for the management of MS, DMT initiation was associated with reductions in disease relapses, hospitalizations, ER/ UC visits, and non-prescription medical costs. Non-prescription medical costs in the post-index period were significantly lower for the DMF, IFN $\beta$, GA, and fingolimod cohorts, with the greatest cost reductions observed in the DMF and fingolimod cohorts. Cost reductions were largely driven by reductions in the use of outpatient services and inpatient hospital visits. Consistent with these cost reductions, ARR reductions during the post-index period were significantly greater in the DMF and fingolimod cohorts than the IFN $\beta$, GA, and teriflunomide cohorts. Hospitalizations and ER/UC visits were lower in the post-index period for all DMT cohorts. Together, these findings indicate that DMT initiation in patients without DMT exposure in the previous year is associated with reductions in non-prescription medical costs, resource utilization, and relapse rates.

The reductions in healthcare resource utilization after DMT initiation observed in this study are consistent with previous analyses of real-world data reporting reductions in inpatient stays and ER visits for patients initiating treatment with a DMT, and for patients with lower disease activity or fewer relapses [26, 27]. In a cohort of patients initiating natalizumab therapy, significant reductions in MS-related inpatient stays (7.6 vs. $2.4 \%, p<0.001)$ and length of hospital stay (7.12 vs. 6.26 days, $p=0.005$ ) were observed in the post-index period compared with the preindex period [18]. Similarly, an analysis of patients with MS and high relapse activity ( $\geq 2$ relapses in the 1-year assessment period) demonstrated increased all-cause ER visits and hospitalizations compared with patients with lower disease activity [26]. Moreover, a review of the impact of DMT adherence reported that increased adherence is associated not only with a lower risk of relapse (odds ratio [OR] $0.71,95 \%$ CI $0.59-0.85$ ) but also a reduction in MS-related hospitalization (OR $0.63,95 \%$ CI $0.47-0.83$ ) and ER visits (OR $0.80,95 \%$ CI $0.60-1.07$ ) [27].

Understanding the drivers of changes in healthcare resource utilization, medical costs, and ARRs is important to enable clinicians and healthcare providers to optimize use of limited healthcare resources while maximizing patient benefit. In the present study, ARR was significantly lower in the year after DMT initiation for the DMF and fingolimod cohorts, consistent with previous studies of DMT effectiveness in routine clinical practice $[16,17]$, and a cost-effectiveness simulation in a French healthcare setting [28]. Reductions in both ARRs and healthcare resource utilization and costs in the DMF and fingolimod cohorts suggest an association between lower ARR and reduced healthcare resource utilization and costs, a finding consistent with previous real-world studies [19, 26, 27]. However, interpretation of these results should take into account the potential confounding effect of determinants of pre-index ARR, such as level of disability, MS phenotype, and time since first MS diagnosis, as these data were not available in the US claims data source. Nevertheless, previous studies have demonstrated an association between reduced relapse rates and reduced medical costs [18, 19]. For example, an analysis of the budget impact of DMF for the management of RRMS in Canada demonstrated a cost reduction of Can\$192 per patient per year due to lower relapse rates, which partially offset drug acquisition costs [19]. Similarly, in an analysis of 1458 patients with MS who initiated natalizumab therapy, mean costs associated with inpatient stays were lower in the post-index period compared with the pre-index period (US\$9784 vs. US\$12,078) [18].

Overall healthcare costs after DMT initiation did increase in the cohorts studied, with DMT drug costs the largest component of post-index period costs. However, the higher overall healthcare costs after DMT initiation were associated with improved patient outcomes, specifically reductions in relapse rates. This is consistent with an analysis of 544 patients with RRMS in Italy who initiated a DMT, in which higher overall healthcare costs were associated with a reduction in EDSS progression; the authors hypothesized that this would lead to a reduction in nonmedical direct and indirect costs [29]. DMT costs in our analysis were lower than the anticipated annual DMT costs, which are typically in the range of US\$55,000-65,000 before manufacturer rebates and contracting discounts [30]. Costs in our study are likely to be lower as patients did not have full adherence to treatment, meaning that some prescriptions may not have been filled throughout the 1-year follow-up period. Our sample also included patients who stopped using the index DMT during the follow-up period, thereby reducing medication costs. Our data therefore show the real-world cost of DMTs to insurance plans and patients when these therapies are used in routine practice.

Although cost reductions in the post-index period were not as pronounced in the IFN $\beta$, GA, and teriflunomide cohorts compared with the DMF and fingolimod cohorts, the magnitude of cost reduction in each DMT cohort was consistent with the observed ARR reduction over the same period. However, reductions in hospitalizations, ER/UC visits, and medical costs were observed for the IFN $\beta$, GA, and teriflunomide cohorts despite non-significant ARR changes in the year after DMT initiation, indicating that 
factors other than ARR reduction may influence resource utilization and costs. This is not unexpected, given the potential for ongoing symptoms associated with MS that may not be classified as explicitly relating to a relapse event but that nevertheless impact on patient daily living, particularly with more progressive disease. Future studies are therefore needed to further explore the interrelationship between healthcare resource utilization, costs, and clinical outcomes for patients with MS.

Understanding the potential clinical and resource utilization impact of initiating a new DMT is increasingly important when healthcare professionals are discussing treatment options with patients. The present findings provide further evidence of the differential impact of different DMTs on outcomes and healthcare resource use, and provide clinicians and patients with additional information on non-clinical outcomes, which may impact treatment choice. Moreover, in an era of patient-centered healthcare, understanding the impact of different DMTs on the potential for hospitalization and ER and UC visits enables clinicians and other healthcare professionals to have informed discussions with patients about treatment options for MS, particularly around the potential impact on daily activities and hospital attendance.

\subsection{Limitations}

The study is subject to a number of the limitations associated with retrospective studies utilizing administrative claims data. Due to data limitations, some clinical features (e.g. EDSS score) were not included in the analysis as these data are not included in the US claims database interrogated here. However, this is partially addressed by the fact that $\mathrm{DiD}$ analysis provides estimates that mitigate the impact of confounding factors that did not change over time (e.g. patient history). Our findings indicate that there may be an effect of pretreatment relapse rate on the ontreatment relapse rate. The findings should therefore be considered in terms of disease parameters that are not available in claims data, such as level of disability, MS phenotype, and time since first MS diagnosis. Other limitations include the inclusion of only DMT initiators who were untreated for at least 1 year. Although the patients included in the analysis had no exposure to DMT in the year before initiation, we were not able to confirm whether they were treatment-naive beyond this period due to data limitations. In addition, the findings from this analysis may not be generalized to patient populations in other healthcare systems, and patients may have transitioned into Medicare coverage during the 1-year follow-up period.

DiD regression relies on an assumption of parallel trends, i.e. that the underlying change in the outcome measured, but not the change due to treatment, is consistent across groups over time. For relapse rates, the analysis assumes similar underlying disease progression, i.e. progression of increasing relapse rates, between groups. As the dataset does not include information on patient disability and EDSS, we were not able to confirm that this assumption is valid.

In this study, we used Poisson regression to control for differences between patient cohorts in age, sex, region of residence, number of relapses during the year before the index date, CCI score, and the presence of individual MSrelated symptoms. While propensity score matching provides an alternative approach for controlling for differences in patient characteristics, we opted to use regression analysis to maintain statistical precision by allowing the use of all patient information, rather than discarding information for unmatched patients [31, 32]. However, further studies are needed to confirm whether the clinical and demographic variables not captured in claims data influence healthcare resource utilization, costs, and relapse rates in patients with MS receiving a DMT.

Patient prior treatment and clinical characteristics in retrospective claims database analyses also have the potential to introduce selection bias. Our study included patients without DMT exposure in the previous year, potentially preferentially selecting patients with less severe disease who may be less likely to receive treatment with fingolimod, which is often given as second-line therapy and/or to patients with greater disease severity. However, in our dataset, fingolimod appears to be prescribed to similar patients as the other DMTs investigated. In the pre-index period, ARRs and the percentage of patients experiencing a relapse were similar for the fingolimod and DMF cohorts, and pre-index hospitalization and ER/UC costs were lower in the fingolimod cohort than the DMF cohort. However, claims data do not include information on patient clinical and disease characteristics, or EDSS score, and the current findings should be interpreted within this context.

Finally, in the absence of clinical evidence of MS relapse from the claims data, we used an algorithm to estimate relapse rates. Relapse episodes were identified based on an algorithm derived from the ICD-9-CM diagnosis code and details of medications dispensed and subsequently validated against medical charts and clinician input [23, 24]. This algorithm has been used in previous studies to determine relapse rates in patients with RRMS based on claims data and allows the identification of moderate-to-severe relapses that result in a clinical encounter; patients with mild relapses may not present clinically and, therefore, rates of mild relapse may be underestimated. Algorithm validation by Chastek and colleagues provided classification of $67.3 \%$ of patients with relapses (positive predictive value) and $70.0 \%$ of patients 
without relapses (negative predictive value) in a sample of 300 patients [24]. While this approach may underestimate the overall relapse incidence, this effect is anticipated to be uniform across all comparisons, and ARRs reported in the present study are consistent with those reported from clinical trials and in other studies [13, 33-35].

\section{Conclusions}

Results from this comparative analysis of real-world healthcare resource utilization, costs, and relapse rates for the new oral DMTs are consistent with previous studies and highlight the positive impact of DMT initiation on healthcare system resource use from a hospital and clinic capacity perspective.

Although reduced healthcare resource utilization associated with improved patient outcomes has the potential to partially offset costs associated with DMT therapy for the management of MS, real-world data on comparative resource utilization and costs in routine clinical practice are limited. This large, retrospective US claims data analysis demonstrates that costs associated with DMT are partially offset by reductions in non-prescription medical costs and healthcare resource utilization in the post-initiation period. Of the DMTs assessed, DMF provided the greatest reduction in non-prescription medical costs. Non-prescription medical cost reductions were predominantly driven by decreased use of outpatient services and by a reduction in inpatient hospital stays. Further research is required to determine the long-term impact of DMT initiation on resource utilization and costs in routine clinical practice.

Acknowledgements Writing and editorial support for preparation of this manuscript was provided by Dr Nick Leach and Dr Daniel Squirrell, OPEN Access Consulting (Marlow, UK). Funding for editorial support was provided by Biogen (Cambridge, MA, USA).

Author Contributions AL, MYH, WSY, JK, and NW conceived, and designed, the study. WSY and NW performed the database analyses. All authors had access to, and were involved in, interpreting the data. JN, AL, and MYH wrote the manuscript with external editorial support from NL and DS. All authors were responsible for critically revising and approving the manuscript at each draft.

\section{Compliance with Ethical Standards}

Conflicts of interest Jacqueline Nicholas has received research funding from Genzyme, Novartis, Teva, Biogen, and Alexion, and has received consulting and speaking honoraria from Genzyme, Novartis, Teva, Biogen, and Medtronic. Aaron Boster has received research funding from Genentech, Actelion, and Mallinckrodt, and has received consulting and speaking honoraria from Genzyme, Novartis, Teva, Biogen, and Medtronic. Monica Fay and Andrew Lee are employees of, and hold stock and/or stock options in, Biogen. Ning
Wu, Wei-Shi Yeh, Jon Kendter, and Ming-Yi Huang were employees of Biogen at the time of the study.

Funding This study was funded by Biogen (Cambridge, MA, USA).

Ethical approval This article does not report data from any studies with human participants or animals performed by any of the authors.

Data Availability The datasets analyzed in the present study are not publicly available because they were licensed with restrictions from a third-party source.

Open Access This article is distributed under the terms of the Creative Commons Attribution-NonCommercial 4.0 International License (http://creativecommons.org/licenses/by-nc/4.0/), which permits any noncommercial use, distribution, and reproduction in any medium, provided you give appropriate credit to the original author(s) and the source, provide a link to the Creative Commons license, and indicate if changes were made.

\section{References}

1. Goldenberg MM. Multiple sclerosis review. Pharm Ther. 2012;37:175-84.

2. Compston A, Coles A. Multiple sclerosis. Lancet Lond Engl. 2008;372:1502-17.

3. Kantarci OH, Pirko I, Rodriguez M. Novel immunomodulatory approaches for the management of multiple sclerosis. Clin Pharmacol Ther. 2014;95:32-44.

4. Campbell J, Ghushchyan V, McQueen R, Cahoon-Metzger S, Livingston T, Vollmer T, et al. Burden of multiple sclerosis on direct, indirect costs and quality of life: national US estimates. Mult Scler Relat Disord. 2014;3:227-36.

5. Browne P, Chandraratna D, Angood C, Tremlett H, Baker C, Taylor BV, et al. Atlas of multiple sclerosis 2013: a growing global problem with widespread inequity. Neurology. 2014;83:1022-4.

6. McKay KA, Kwan V, Duggan T, Tremlett H. Risk factors associated with the onset of relapsing-remitting and primary progressive multiple sclerosis: a systematic review. Biomed Res Int. 2015;2015:817238.

7. Adelman G, Rane SG, Villa KF. The cost burden of multiple sclerosis in the United States: a systematic review of the literature. J Med Econ. 2013;16:639-47.

8. Julian L, Vella L, Vollmer T, Hadjimichael O, Mohr D. Employment in multiple sclerosis: exiting and re-entering the work force. J Neurol. 2008;255:1354-60.

9. Ivanova JI, Birnbaum HG, Samuels S, Davis M, Phillips AL, Meletiche D. The cost of disability and medically related absenteeism among employees with multiple sclerosis in the US. Pharmacoeconomics. 2009;27:681-91.

10. Oh J, O'Connor PW. Established disease-modifying treatments in relapsing-remitting multiple sclerosis. Curr Opin Neurol. 2015;28:220-9.

11. Cohen BA, Coyle PK, Leist T, Oleen-Burkey MA, Schwartz M, Zwibel $\mathrm{H}$. Therapy optimization in multiple sclerosis: a cohort study of therapy adherence and risk of relapse. Mult Scler Relat Disord. 2015;4:75-82.

12. Hutchinson M, Fox RJ, Havrdova E, Kurukulasuriya NC, Sarda SP, Agarwal S, et al. Efficacy and safety of BG-12 (dimethyl fumarate) and other disease-modifying therapies for the treatment of relapsing-remitting multiple sclerosis: a systematic review and 
mixed treatment comparison. Curr Med Res Opin. 2014;30:613-27.

13. Tramacere I, Del Giovane C, Salanti G, D’Amico R, Filippini G. Immunomodulators and immunosuppressants for relapsingremitting multiple sclerosis: a network meta-analysis. Cochrane Database Syst Rev. 2015;9:CD011381.

14. Hannan EL. Randomized clinical trials and observational studies: guidelines for assessing respective strengths and limitations. JACC Cardiovasc Interv. 2008;1:211-17.

15. Kennedy-Martin T, Curtis S, Faries D, Robinson S, Johnston J. A literature review on the representativeness of randomized controlled trial samples and implications for the external validity of trial results. Trials. 2015;16:495.

16. Hersh CM, Love TE, Cohn S, Hara-Cleaver C, Bermel RA, Fox RJ, et al. Comparative efficacy and discontinuation of dimethyl fumarate and fingolimod in clinical practice at 12-month followup. Mult Scler Relat Disord. 2016;10:44-52.

17. Boster A, Nicholas J, Wu N, Yeh W-S, Fay M, Edwards M, et al. Comparative effectiveness research of disease-modifying therapies for the management of multiple sclerosis: analysis of a large health insurance claims database. Neurol Ther. 2017;6:91-102.

18. Bonafede MM, Johnson BH, Watson C. Health care-resource utilization before and after natalizumab initiation in multiple sclerosis patients in the US. Clinicoecon Outcomes Res. 2013;6:11-20.

19. Dorman E, Kansal AR, Sarda S. The budget impact of introducing delayed-release dimethyl fumarate for treatment of relapse-remitting multiple sclerosis in Canada. J Med Econ. 2015;18:1085-91.

20. Charlson ME, Pompei P, Ales KL, MacKenzie CR. A new method of classifying prognostic comorbidity in longitudinal studies: development and validation. J Chronic Dis. 1987;40:373-83.

21. Curkendall SM, Wang C, Johnson BH, Cao Z, Preblick R, Torres AM, et al. Potential health care cost savings associated with early treatment of multiple sclerosis using disease-modifying therapy. Clin Ther. 2011;33:914-25.

22. Consumer Price Index. Bureau of labor statistics. http://www.bls. gov/cpi/. Accessed May 2016.

23. Ollendorf DA, Jilinskaia E, Oleen-Burkey M. Clinical and economic impact of glatiramer acetate versus beta interferon therapy among patients with multiple sclerosis in a managed care population. J Manag Care Pharm. 2002;8:469-76.
24. Chastek BJ, Oleen-Burkey M, Lopez-Bresnahan MV. Medical chart validation of an algorithm for identifying multiple sclerosis relapse in healthcare claims. J Med Econ. 2010;13:618-25.

25. Donald SG, Lang K. Inference with difference-in-differences and other panel data. Rev Econ Stat. 2007;89:221-33.

26. Raimundo K, Tian H, Zhou H, Zhang X, Kahler KH, Agashivala $\mathrm{N}$, et al. Resource utilization, costs and treatment patterns of switching and discontinuing treatment of MS patients with high relapse activity. BMC Health Serv Res. 2013;13:131.

27. Lizán L, Comellas M, Paz S, Poveda JL, Meletiche DM, Polanco C. Treatment adherence and other patient-reported outcomes as cost determinants in multiple sclerosis: a review of the literature. Patient Prefer Adher. 2014;8:1653-64.

28. Chevalier J, Chamoux C, Hammès F, Chicoye A. Cost-effectiveness of treatments for relapsing remitting multiple sclerosis: a French societal perspective. PLoS One. 2016;11:e0150703.

29. Moccia M, Palladino R, Lanzillo R, Carotenuto A, Russo CV, Triassi M, et al. Healthcare costs for treating relapsing multiple sclerosis and the risk of progression: a retrospective Italian cohort study from 2001 to 2015. PLoS One. 2017;12:e0169489.

30. Hartung DM, Bourdette DN, Ahmed SM, Whitham RH. The cost of multiple sclerosis drugs in the US and the pharmaceutical industry: too big to fail? Neurology. 2015;84:2185-92.

31. Pearce N. Analysis of matched case-control studies. BMJ. 2016;352:1969.

32. Klein JP, Rizzo JD, Zhang MJ, Keiding N. Statistical methods for the analysis and presentation of the results of bone marrow transplants. Part 2: regression modeling. Bone Marrow Transplant. 2001;28:1001-11.

33. Bergvall N, Makin C, Lahoz R, Agashivala N, Pradhan A, Capkun $\mathrm{G}$, et al. Relapse rates in patients with multiple sclerosis switching from interferon to fingolimod or glatiramer acetate: a US claims database study. PLoS One. 2014;9:e88472.

34. Kalincik T, Jokubaitis V, Izquierdo G, Duquette P, Girard M, Grammond $\mathrm{P}$, et al. Comparative effectiveness of glatiramer acetate and interferon beta formulations in relapsing-remitting multiple sclerosis. Mult Scler. 2015;21:1159-71.

35. Bergvall N, Makin C, Lahoz R, Agashivala N, Pradhan A, Capkun $\mathrm{G}$, et al. Comparative effectiveness of fingolimod versus interferons or glatiramer acetate for relapse rates in multiple sclerosis: a retrospective US claims database analysis. Curr Med Res Opin. 2013;29:1647-56. 